\title{
Higgs production cross-section in a Standard Model with four generations at the LHC
}

\author{
Charalampos Anastasiou ${ }^{1}$, Stephan Buehler ${ }^{1}$, Elisabetta Furlan ${ }^{2}$, Franz Herzog ${ }^{1}$, Achilleas Lazopoulos ${ }^{1}$ \\ ${ }^{1}$ Institute of Theoretical Physics, ETH Zurich, 8093 Zurich, Switzerland \\ ${ }^{2}$ Physics Department, Brookhaven National Laboratory, Upton, NY 11973, USA
}

\begin{abstract}
We present theoretical predictions for the Higgs boson production cross-section via gluon fusion at the LHC in a Standard Model with four generations. We include QCD corrections through NLO retaining the full dependence on the quark masses, and the NNLO corrections in the heavy quark effective theory approximation. We also include electroweak corrections through three loops. Electroweak and bottom-quark contributions are suppressed in comparison to the Standard Model with three generations.
\end{abstract}

\section{Introduction}

The Tevatron and the LHC are becoming increasingly sensitive to potential signals of a Standard Model (SM) Higgs boson. The highest sensitivity is achieved for a Higgs boson of mass $m_{h} \sim 2 m_{W}$, where the branching ratio of the Higgs decaying into a pair of $W$ bosons is close to one.

The most significant production channel for these searches is gluon fusion. This is a loopmediated process, and in the Standard Model the corresponding amplitudes are dominated by top-quark loops. Gluon fusion may be sensitive to new coloured, heavy particles with a large coupling to the Higgs boson. Limits on the Higgs production cross-section from ongoing experimental searches are model-specific, and a dedicated theoretical prediction to Standard Model extensions is often required.

A simple extension of the Standard Model may include a fourth family of quarks and leptons. This model is theoretically constrained to not very large fourth-generation quark masses, in order to preserve perturbativity 1 . To evade limits from precision electroweak tests, the mass difference of the fourth-generation quarks is also restricted to be small 2 . Finally, the fourth-generation neutrino is required to have a mass greater than half the mass of the Z-boson from the LEP limits on the invisible Z-boson decay width.

One might find little theoretical appeal in a naive Standard Model with four generations. As in the Standard Model, it suffers from the hierarchy problem, and in addition it introduces a rather awkwardly heavy neutrino. Nevertheless, its predictions are rather spectacular in modifying the Higgs boson cross-section at hadron colliders and can be tested easily with Tevatron and early LHC data. The Tevatron has published limits on the Higgs boson cross-section in this model, excluding a wide range of Higgs boson masses 3 . Recently the CMS collaboration carried out a similar study 4 .

A precise calculation of the Higgs boson gluon fusion cross-section has been made in Ref. 5 , where numerical results have been given for the Tevatron. In this work, we compute the cross- 
section at the LHC with center of mass energy $\sqrt{s}=7 \mathrm{TeV}$.

\section{Calculation details}

In the Standard Model, gluon fusion Higgs production is mediated by massive quarks and electroweak gauge bosons. The dominant contribution is given by top-quark loops. Bottomquark loops and $W, Z$ loops yield a small contribution, and quarks lighter than the bottom can be neglected.

The gluon fusion cross-section through massive quark loops has been computed through NLO in perturbative QCD both in the heavy quark effective theory 617 and retaining the full top-mass dependence 89 . The NLO corrections are large, motivating the calculation of the crosssection through NNLO in perturbative QCD 10/11/12]. The NNLO corrections have first been computed in the heavy quark effective theory (HQET). Recently, subleading corrections in the inverse top-quark mass expansion have been calculated $13 \mid 14$, validating the quality of the HQET approximation. Other corrections include the exact two-loop electroweak contributions 15 and mixed QCD and electroweak corrections 16 using an effective theory approach.

A precise prediction of the Higgs boson cross-section in fixed order perturbation theory may be obtained by combining the above contributions, with an estimated uncertainty of about $\pm 10 \%$ due to missing higher order effects. Calculations resumming factorizable contributions of infrared nature are in agreement with this error uncertainty $17 \mid 18$. A similar uncertainty at the LHC and slightly larger at the Tevatron is due to parton distribution functions.

In this work, we compute the cross-section in a Standard Model with four fermion generations. The following points make this calculation different from the calculation in the Standard Model.

- The gluon fusion amplitudes receive contributions from the up and down quarks of the fourth generation. We compute these contributions through NLO retaining the exact mass dependence. In the strict HQET limit, the cross-section is increased by a factor of nine with respect to the SM cross-section.

- Bottom-quark contributions are suppressed with respect to the SM by roughly a factor of three. We compute bottom-quark contributions exactly through NLO in perturbative QCD.

- Through NLO, the Wilson coefficient of the HQET operator is three times the Standard Model Wilson coefficient. At NNLO, the Wilson coefficient depends on the masses of the the heavy quarks and it also receives contributions from three-loop diagrams containing two different heavy quarks 5 .

- Two-loop electroweak corrections due to light quarks are given by the same diagrams as in the Standard Model 19 . They predominate the electroweak corrections for a light Higgs boson. They are suppressed in the Standard Model with four generations compared to the three-generation SM. In the SM, additional electroweak corrections due to the top quark are very small for a light Higgs boson and have been computed in Ref. 15 . Here we include the full two-loop Standard Model electroweak corrections of Ref. 15 , but we neglect the two-loop electroweak corrections with quarks of the fourth generation.

We compute the NNLO corrections in the effective theory approximation. We normalize these corrections to the Born cross-section with the exact mass dependence on the masses of the top-quark and the quarks of the fourth generation. We do not include bottom-quark loops at NNLO. 


\section{Predictions for the gluon fusion cross-section for $\sqrt{s}=7 \mathrm{TeV}$}

In this section we present our numerical results for the gluon fusion cross-section in a Standard Model with four generations at the current LHC energy of $7 \mathrm{TeV}$ and for Higgs boson masses in the range $m_{h} \in[110 \mathrm{GeV}, 300 \mathrm{GeV}]$. The parameter space of the Standard Model with four generations is quite limited. The model becomes non-perturbative for values of the heavy quark masses roughly above $\sim 500 \mathrm{GeV}^{1}$. A large mass splitting of the quarks of the fourth generation induces large corrections to oblique electroweak parameters 2 . To comply with these constraints we present the cross-section for two scenarios, where the mass of the fourth-generation down quark is chosen as

$$
\text { scenario 1: } m_{d_{4}}=300 \mathrm{GeV}, \quad \text { scenario } 2: m_{d_{4}}=400 \mathrm{GeV} \text {. }
$$

The mass of the fourth-generation up quark given by 2

$$
m_{u_{4}}-m_{d_{4}}=50 \mathrm{GeV}+10 \mathrm{GeV} \times \log \left(\frac{m_{h}}{115 \mathrm{GeV}}\right) \mathrm{GeV} .
$$

For the top- and bottom-quark masses we take

$$
m_{t}=172 \mathrm{GeV}, \quad m_{b}\left(m_{b}\right)=4.2 \mathrm{GeV} .
$$

We use the MSTW08 NNLO parton densitities 20 and quote the " $\alpha_{s}+$ pdf" uncertainty at the 90\% confidence level (CL). To estimate the uncertainty due to higher order perturbative effects, we vary the renormalization and factorization scales in the range $\frac{m_{h}}{4} \leq \mu_{r}=\mu_{f} \leq m_{h}$. The scale variation and pdf uncertainty are very similar to the equivalent uncertainties in the Standard Model. We present our results in Table 1 .

\section{Sensitivity to parton distributions}

Besides the MSTW08 pdf set, other two NNLO parton distribution sets are currently made available by the GJR 21 and $\mathrm{ABKM}^{22}$ collaborations. In Table 2 we present the central value of the cross-section for $\mu=\frac{m_{h}}{2}$, the scale variation uncertainty and the pdf uncertainty (at the $68 \% \mathrm{CL})$ for $m_{h}=110,165,200,300 \mathrm{GeV}$ and the ABKM09, GJR and MSTW08 pdf sets.

We find that the GJR and ABKM09 pfds give central values for the cross-section which can be up to $12 \%$ smaller than the one of MSTW08. These differences are larger than what anticipated from the quoted parton density uncertainties at the $68 \%$ confidence level. The MSTW08 provides uncertainties at the 90\% confidence level, which yield about twice as large an uncertainty for the gluon fusion cross-section. These overlap, albeit marginally for lower Higgs boson masses, with the uncertainties of GJR and ABKM09.

All three groups provide consistent determinations of the parton distributions with selfconsistent choices and assumptions, and there is no "bullet-proof" argument to choose one over the others. Nevertheless, we prefer MSTW08 as our default pdf. Our main reasons for this choice are that MSTW08 includes jet data directly sensitive to the gluon density and that their central value of $\alpha_{s}\left(m_{Z}\right)$ is in very good agreement with the world average 23 and determinations

from jet data at $e^{+} e^{-}$colliders using NNLO jet cross-sections 24 . We also find it prudent to estimate the pdf uncertainty of the cross-section using parton density uncertainties at the $90 \%$ CL. The extraction of parton distribution functions will soon be assisted with early LHC data, which we hope will help to resolve the discrepances among the various pdf sets. 


\begin{tabular}{|c|c|c|c|c|c|c|}
\hline $\mathrm{GeV}$ & $\begin{array}{c}\sigma[p b] \\
\text { scenario } 1\end{array}$ & $\begin{array}{c}\sigma[p b] \\
\text { scenario } 2\end{array}$ & $\begin{array}{cc}\delta^{(+)}\left(\mathrm{pdf}+\alpha_{s}\right) & \% \\
\text { MSTW08 } \\
90 \% \mathrm{CL} & \\
90 \% & \end{array}$ & $\begin{array}{cc}\delta^{(-)}\left(\mathrm{pdf}+\alpha_{s}\right) & \\
\text { MSTWOo8 } & \% \\
90 \% \mathrm{CL} & \end{array}$ & $\delta^{(+)}(\mu) \%$ & $\delta^{(-)}(\mu) \%$ \\
\hline 105 & 202.33 & 201.39 & 7.9 & -7.6 & 9.2 & -9.7 \\
\hline 110 & 183.41 & 182.51 & 7.9 & -7.6 & 9.0 & -9.7 \\
\hline 115 & 166.85 & 165.97 & 7.9 & -7.6 & 8.9 & -9.6 \\
\hline 120 & 152.27 & 151.41 & 7.9 & -7.6 & 8.7 & -9.6 \\
\hline 125 & 139.38 & 138.54 & 7.9 & -7.6 & 8.6 & -9.6 \\
\hline 130 & 127.93 & 127.12 & 7.9 & -7.6 & 8.5 & -9.5 \\
\hline 135 & 117.72 & 116.93 & 7.9 & -7.6 & 8.4 & -9.5 \\
\hline 140 & 108.59 & 107.81 & 7.9 & -7.6 & 8.3 & -9.5 \\
\hline 145 & 100.39 & 99.628 & 7.9 & -7.6 & 8.2 & -9.4 \\
\hline 150 & 93.002 & 92.253 & 7.9 & -7.6 & 8.1 & -9.4 \\
\hline 155 & 86.298 & 85.563 & 7.9 & -7.6 & 8.0 & -9.4 \\
\hline 160 & 80.091 & 79.371 & 7.9 & -7.6 & 7.9 & -9.4 \\
\hline 165 & 74.221 & 73.516 & 7.9 & -7.7 & 7.8 & -9.4 \\
\hline 170 & 68.920 & 68.228 & 8.0 & -7.7 & 7.8 & -9.3 \\
\hline 175 & 64.249 & 63.570 & 8.0 & -7.7 & 7.7 & -9.3 \\
\hline 180 & 60.000 & 59.333 & 8.0 & -7.7 & 7.6 & -9.3 \\
\hline 185 & 56.080 & 55.424 & 8.0 & -7.8 & 7.6 & -9.3 \\
\hline 190 & 52.493 & 51.849 & 8.1 & -7.8 & 7.5 & -9.3 \\
\hline 195 & 49.246 & 48.612 & 8.1 & -7.8 & 7.4 & -9.3 \\
\hline 200 & 46.306 & 45.681 & 8.1 & -7.9 & 7.4 & -9.2 \\
\hline 205 & 43.620 & 43.005 & 8.1 & -7.9 & 7.3 & -9.2 \\
\hline 210 & 41.153 & 40.546 & 8.2 & -7.9 & 7.3 & -9.2 \\
\hline 215 & 38.878 & 38.279 & 8.2 & -8.0 & 7.2 & -9.2 \\
\hline 220 & 36.776 & 36.185 & 8.2 & -8.0 & 7.2 & -9.2 \\
\hline 225 & 34.832 & 34.249 & 8.3 & -8.0 & 7.2 & -9.2 \\
\hline 230 & 33.031 & 32.454 & 8.3 & -8.1 & 7.1 & -9.2 \\
\hline 235 & 31.357 & 30.788 & 8.3 & -8.1 & 7.1 & -9.2 \\
\hline 240 & 29.805 & 29.242 & 8.3 & -8.1 & 7.0 & -9.2 \\
\hline 245 & 28.357 & 27.800 & 8.4 & -8.2 & 7.0 & -9.1 \\
\hline 250 & 27.009 & 26.459 & 8.4 & -8.2 & 7.0 & -9.1 \\
\hline 255 & 25.751 & 25.206 & 8.5 & -8.3 & 6.9 & -9.1 \\
\hline 260 & 24.577 & 24.038 & 8.5 & -8.3 & 6.9 & -9.1 \\
\hline 265 & 23.480 & 22.945 & 8.5 & -8.3 & 6.9 & -9.1 \\
\hline 270 & 22.455 & 21.926 & 8.6 & -8.4 & 6.8 & -9.1 \\
\hline 275 & 21.495 & 20.970 & 8.6 & -8.4 & 6.8 & -9.1 \\
\hline 280 & 20.598 & 20.078 & 8.6 & -8.4 & 6.8 & -9.1 \\
\hline 285 & 19.756 & 19.241 & 8.7 & -8.5 & 6.7 & -9.1 \\
\hline 290 & 18.969 & 18.457 & 8.7 & -8.6 & 6.7 & -9.1 \\
\hline 295 & 18.232 & 17.725 & 8.8 & -8.6 & 6.7 & -9.1 \\
\hline 300 & 17.541 & 17.037 & 8.8 & -8.6 & 6.7 & -9.1 \\
\hline
\end{tabular}

Table 1: Gluon fusion cross-section in a Standard Model with four fermion generations. The masses of the fourth generation quarks are chosen according to "scenario 1" and "scenario 2". All cross-sections are computed with $0.1 \%$ Monte-Carlo integration error or better. 


\begin{tabular}{|c|c|c|c|c|}
\hline$\sigma[p b]$ & ABKM09 & GJR & $\mathrm{MSTW08}_{68 \% \mathrm{CL}}$ & MSTW08| $\left.\right|_{90 \% \mathrm{CL}}$ \\
\hline$m_{h}=110 \mathrm{GeV}$ & $167.59 \pm 3.0 \% \mathrm{pdf}$ & $162.78 \pm 3.6 \% \mathrm{pdf}$ & $183.41 \begin{array}{l}+4.0 \\
-3.1\end{array} \% \mathrm{pdf}$ & $\begin{array}{l}+7.9 \% \text { pdf } \\
-7.6 \%\end{array}$ \\
\hline$m_{h}=165 \mathrm{GeV}$ & $66.130 \pm 3.3 \% \mathrm{pdf}$ & $67.713 \pm 3.3 \%$ pdf & $74.221 \stackrel{+4.0}{-3.3} \%$ pdf & $\begin{array}{l}+7.9 \% \text { pdf } \\
-7.7 \%\end{array}$ \\
\hline$m_{h}=200 \mathrm{GeV}$ & $40.634 \pm 3.6 \%$ pdf & $42.867 \pm 3.5 \%$ pdf & $46.306 \stackrel{+4.1}{-3.4} \%$ pdf & $\begin{array}{l}+8.1 \% \text { pdf } \\
-7.9\end{array}$ \\
\hline$m_{h}=300 \mathrm{GeV}$ & $14.768 \pm 4.7 \%$ pdf & $16.786 \pm 5.0 \% \mathrm{pdf}$ & $17.541_{-3.9}^{+4.3} \% \mathrm{pdf}$ & $\begin{array}{l}+8.8 \% \text { pdf } \\
-8.6\end{array}$ \\
\hline
\end{tabular}

Table 2: A comparison for the gluon fusion cross-section in the "scenario 1" of the four-generation Standard Model with the three available NNLO pdf sets: ABKM09, GJR and MSTW08.

\section{Branching ratios}

For a complete prediction of a Higgs signal cross-section at colliders the branching ratios of the Higgs boson decays to observable final states are needed. Branching ratios are significantly modified with respect to the Standard Model when adding a fourth quark and lepton generation.

In this model, the partial decay width of the Higgs boson to gluons is enhanced by a large factor which reaches nine in the HQET limit. This width dominates for a light Higgs boson. The decay widths to $W W$ and $Z Z$ are significant for a range of values of the Higgs boson mass above $140 \mathrm{GeV}$. However, the corresponding branching ratios are smaller than in the Standard Model. These decays are important, given that the Tevatron and the LHC are quite sensitive and their experimental study may lead to a Higgs boson discovery with a modest amount of data. In the four-generation SM, novel decays of a heavy Higgs boson to the leptons of the fourth generation emerge and assume a significant width.

A systematic study of the Higgs boson decays has been made in Ref. 2 , where the branching ratios have been computed by modifying the program HDECAY 25 . Tabulated results from Ref. 2 for the branching ratios of the Higgs boson, corresponding to "scenario 1" and "scenario 2" of our study, can be found in Ref. $\underline{3}$.

\section{Conclusions}

In this work we have studied the Higgs boson cross-section at the LHC, in a Standard Model with a fourth generation. We have computed the cross-section through NNLO in perturbative QCD, including finite quark-mass effects and electroweak corrections through NLO. We have provided an estimate of the uncertainty due to higher order perturbative effects, and have studied the sensitivity of the cross-section on various NNLO parameterizations of the parton densities and their uncertainties. Our results are of direct relevance to the ongoing studies for the discovery of the Higgs boson at the LHC.

\section{Acknowledgments}

Research supported by the Swiss National Foundation under contract SNF 200020-126632 and the DOE under Grant DE-AC02-98CH10886.

\section{References}

1. W. J. Marciano, G. Valencia and S. Willenbrock, Phys. Rev. D 40, 1725 (1989).

2. G. D. Kribs, T. Plehn, M. Spannowsky and T. M. P. Tait, Phys. Rev. D 76, 075016 (2007) arXiv:0706.3718 [hep-ph]]. 
3. T. Aaltonen et al. [CDF and D0 Collaboration], arXiv:1005.3216 [hep-ex].

4. S. Chatrchyan et al. [CMS Collaboration], arXiv:1102.5429 [hep-ex].

5. C. Anastasiou, R. Boughezal and E. Furlan, JHEP 1006, 101 (2010) arXiv:1003.4677 [hep-ph]].

6. S. Dawson, Nucl. Phys. B 359, 283 (1991).

7. A. Djouadi, M. Spira and P. M. Zerwas, Phys. Lett. B 264, 440 (1991).

8. D. Graudenz, M. Spira and P. M. Zerwas, Phys. Rev. Lett. 70, 1372 (1993).

9. M. Spira, A. Djouadi, D. Graudenz and P. M. Zerwas, Nucl. Phys. B 453, 17 (1995) arXiv:hep-ph/9504378.

10. R. V. Harlander and W. B. Kilgore, Phys. Rev. Lett. 88, 201801 (2002) arXiv:hep-ph/0201206.

11. C. Anastasiou and K. Melnikov, Nucl. Phys. B 646, 220 (2002) [arXiv:hep-ph/0207004.

12. V. Ravindran, J. Smith and W. L. van Neerven, Nucl. Phys. B 665, 325 (2003) arXiv:hep-ph/0302135].

13. R. V. Harlander and K. J. Ozeren, JHEP 0911, 088 (2009) arXiv:0909.3420 [hep-ph]].

14. A. Pak, M. Rogal and M. Steinhauser, JHEP 1002, 025 (2010) arXiv:0911.4662 [hep-ph]].

15. S. Actis, G. Passarino, C. Sturm and S. Uccirati, Phys. Lett. B 670, 12 (2008) arXiv:0809.1301 [hep-ph]].

16. C. Anastasiou, R. Boughezal and F. Petriello, JHEP 0904, 003 (2009) arXiv:0811.3458 [hep-ph]].

17. S. Catani, D. de Florian, M. Grazzini and P. Nason, JHEP 0307, 028 (2003) arXiv:hep-ph/0306211.

18. D. de Florian and M. Grazzini, Phys. Lett. B 674, 291 (2009) [arXiv:0901.2427 [hep-ph]].

19. U. Aglietti, R. Bonciani, G. Degrassi and A. Vicini, Phys. Lett. B 595, 432 (2004) arXiv:hep-ph/0404071].

20. A. D. Martin, W. J. Stirling, R. S. Thorne and G. Watt, Eur. Phys. J. C 63, 189 (2009) arXiv:0901.0002 [hep-ph]].

21. P. Jimenez-Delgado and E. Reya, Phys. Rev. D 80, 114011 (2009) arXiv:0909.1711 [hep$\mathrm{ph}]]$.

22. S. Alekhin, J. Blumlein, S. Klein and S. Moch, Phys. Rev. D 81, 014032 (2010) arXiv:0908.2766 [hep-ph]].

23. S. Bethke, Eur. Phys. J. C 64, 689 (2009) arXiv:0908.1135 [hep-ph]].

24. G. Dissertori, A. Gehrmann-De Ridder, T. Gehrmann, E. W. N. Glover, G. Heinrich and H. Stenzel, Phys. Rev. Lett. 104, 072002 (2010) arXiv:0910.4283 [hep-ph]].

25. A. Djouadi, J. Kalinowski and M. Spira, Comput. Phys. Commun. 108, 56 (1998) arXiv:hep-ph/9704448.

26. C.D. Buchanan et al, Phys. Rev. D 45, 4088 (1992). 